\title{
Protesi o salute?
}

\section{Prosthesis or health?}

Giovanni Lodi

Dipartimento di Scienze Biomediche, Chirurgiche e Odontoiatriche

Università degli Studi di Milano

via Beldiletto 1/3 Milano 20142

Italia

giovanni.lodi@unimi.it

tel. + 390250319021

fax. +390250319041

The Lancet, una delle due o tre più importanti riviste di medicina al mondo, ha dedicato qualche mese fa diverse pagine alla salute della bocca: evento inconsueto che quindi merita attenzione. Lo fa con due articoli piuttosto corposi. Nel primo viene presentato lo stato di salute orale globale, con dati capaci di impressionare anche agli addetti ai lavori: uno per tutti i 3 miliardi e mezzo di individui che globalmente soffrono di carie, malattia parodontale ed edentulismo. Metà dell'umanità tutta.

Ma è il secondo articolo che affonda il colpo, portando una critica radicale all'intero sistema: "I'odontoiatria del 21esimo secolo è stata incapace di affrontare la sfida mondiale delle malattie della bocca". Secondo gli autori il peccato originale del modello odontoiatrico attuale è di essere troppo focalizzato sui trattamenti. Trattamenti non finalizzati alla cura delle malattie, ma alla riparazione dei danni che provocano.

Trattamenti sempre più specialistici e ad alto contenuto tecnologico, ritenuti da qualsiasi sistema sanitario pubblico non essenziali oltre che troppo costosi, risultando inaccessibili per (quasi) tutti coloro ne avrebbero maggiormente bisogno.

Modello su cui sono fondati i corsi di laurea in odontoiatria di tutto il mondo, che quindi lo preservano immutato (e in effetti, se ci pensate, il nostro corso di laurea è in odontoiatria e protesi dentaria e non, che so, odontoiatria e salute orale).

Neanche la prevenzione come l'abbiamo fatta fino a ora viene risparmiata dalle critiche. Va benissimo insegnare l'igiene orale e l'educazione alimentare ai nostri pazienti, scrivono i colleghi, ma se non si interviene a livello di popolazione contrastando i determinanti delle malattie, lo zucchero in primis, con azioni simili a quelle attuate contro il fumo, la partita è persa in partenza.

Queste sono solo alcune delle questioni messe sul tavolo da un testo che non necessita di essere condiviso in toto, ma la cui lettura sarebbe da prescrivere a chiunque si occupi di salute della bocca.

E intanto i colleghi che si occupano di odontoiatria pediatrica (la SIOI), un importante istituto di ricerca (il Mario Negri) e uno di indagini statistiche (la DOXA), ci riferiscono di dati preoccupanti sulla malattia cariosa tra i piccoli italiani.

Buona lettura, 